\title{
Motion of a sphere through a polymer solution
}

\author{
Tai-Hsi Fan, ${ }^{1}$ Jan K. G. Dhont, ${ }^{2}$ and Remco Tuinier ${ }^{2}$ \\ ${ }^{1}$ Department of Mechanical Engineering, University of Connecticut, Storrs, Connecticut 06269-3139, USA \\ ${ }^{2}$ Forschungszentrum Jülich, Institut für Festkörperforschung, 52425 Jülich, Germany
}

(Received 25 July 2006; published 19 January 2007)

\begin{abstract}
We present analytical solutions of the hydrodynamic resistance force a spherical particle experiences when it moves through a polymer solution containing nonadsorbing chains. Polymer depletion results in a reduced polymer concentration and fluid viscosity near the particle surface. The nonuniform physical properties in the fluid phase affect the transport behavior of a translating and rotating sphere as compared with the case of uniform properties. Based on Stokes' stream function theory, we develop a simplified two-layer approximation by using a step function to represent the viscosity profile. The presence of the polymer solution is formulated in terms of correction functions to the translational and rotational friction of a sphere in a pure solvent. The results are in fair agreement with systematic measurements of the friction of a colloidal sphere when it moves through a polymer solution [Koenderink et al., Phys. Rev. E 69, 021804 (2004)]. The analysis also predicts an apparent slip length in terms of the viscosity ratio and thickness of the depletion layer.
\end{abstract}

DOI: 10.1103/PhysRevE.75.011803

PACS number(s): 61.25.Hq, 66.20.+d, 82.70.Dd

\section{INTRODUCTION}

In many biological processes proteins move through media consisting of concentrated biopolymer (including for instance DNA and/or polysaccharide) solutions [1]. Studying the effect of the presence of nonadsorbing polymers on protein transport therefore helps to better understand biological systems. In colloid physics, the diffusion [2-8] and sedimentation $[9,10]$ behaviors of colloids through a solution containing nonadsorbing polymer chains are of fundamental interest, but the complex physics involved are not yet fully understood [11]. Analyzing the flow of a colloid through a complex medium is essential for interpreting the so-called microrheological measurements using optical techniques; see, for instance, [12-14] and the extensive review by Waigh [15]. Over several decades the diffusion of colloidal particles through polymer solutions were studied experimentally by various groups $[2-7,16,17]$. The translational self-diffusion coefficient of dilute colloidal suspensions in a polymer solution was measured by dynamic light scattering (quasielastic light scattering spectroscopy). In the situation when polymer chains in solution hardly scatter light as compared to the colloidal particles, the fluctuations in the scattered intensity are dominated by particle motions. A resulting correlation function decays as a function of time, from which one can deduce a translational diffusion coefficient [18]. In a depolarized mode one can also analyze the rotational diffusion behavior of the colloid $[17,18]$. Phillies and co-workers (see, for instance, [2,3]) have studied the translational selfdiffusion of well-defined colloidal spheres through polymer solutions, showing that interpretation of the measured friction coefficient of the particles is fairly complicated. For a spherical particle that moves through a medium containing small solvent molecules, the friction coefficient is proportional to the solvent viscosity. If the solvent is replaced by a polymer solution one may naively expect that the friction coefficient is proportional to the viscosity of polymer solution. However, measurements (see, for instance, [7]) indicate that this is only true if the chains are very small compared to the size of the particle. For polymer chains that have a size of the order of the particle radius the apparent or effective viscosity a sphere experiences lies somewhere between the viscosities of solvent and polymer solution. A similar finding was also reported for the rotational diffusion of colloidal particles [17]. Recently, translational diffusion of dilute spheres through a suspension of rods was studied both theoretically and experimentally $[19,20]$. For small spheres compared to the rods the friction that is experienced is determined by the solvent viscosity, whereas the friction clearly increases as the spheres are relatively larger.

Several attempts were made to theoretical understanding of the observed effects of an effective viscosity of a diffusing sphere [6,21-23]. Cukier [21] derived scaling relations for the translational diffusion of a spherical particle through a semidilute polymer solution, while Ngai and Phillies [22] proposed a coupling model for the polymer dynamics that explains the deviation of the effective viscosity from the bulk viscosity. Donath et al. [6] assumed that the no-slip boundary condition is invalid for the case when polymer depletion layer surrounds the moving particle. They modified the boundary condition and predict that the frictional force is reduced by a factor of $2 / 3$ compared to the case with a perfect no-slip condition. In the so-called protein limit, where the size of the particle is much smaller than the characteristic length scale of polymers in solution, the transport properties of protein in semidilute polymer solutions were estimated in terms of a retardation factor [23]. Only a few theoretical studies address the rotational diffusion $[17,24]$ with polymer depletion effect, and the influence of a viscosity gradient on the friction a sphere experiences due to the polymer depletion was not yet considered.

From a phenomenological point of view, it is reasonable to assume that the measured effective viscosity should lie in between the corresponding solvent and polymer solution viscosities. Due to a loss of configurational entropy near the wall, the polymer segment density of the nonadsorbing polymer chains gradually increases from a vanishing value at the particle surface to the bulk value located far away from the particle, resulting in an effective depletion layer [25-30] 
which excludes the centers of mass of the polymer chains. Within this depletion layer, the viscosity is expected to follow the polymer density distribution [31] and gradually increases from the solvent viscosity at the solid surface to the bulk viscosity in the polymer solution. Therefore, when a particle diffuses, the hydrodynamic resistance force lies in between the limiting cases characterized by the viscosities of solvent and polymer solution. Tuinier and Taniguchi [31] derived an expression for the velocity profiles of a nonadsorbing polymer solution near a flat wall when a low shear rate is applied and the characteristic time scale of the fluid flow is much slower than the polymer relaxation time. They suggested the polymer segment density profile is related to viscosity gradients, leading to a quantitative explanation of apparent slip effects. In general, a nonuniform viscosity generates slip effects [32-34], which can be detected using several experimental methods [35], and this slip behavior is expected to appear when a particle moves through a polymer solution.

In this paper we present analytical expressions for the polymer depletion effect on diffusion in terms of the resistance force acting on a translating or a rotating spherical particle. Using a simplified two-layer approximation we derive expressions for the effective viscosity or effective friction coefficient in translational and rotational motions corresponding to the Stokes and Stokes-Einstein-Debye theories, respectively. Note that the idea of using different viscosities can be traced back to Boussinesq's works, collected in [36]. Comparing with experimental results we found that the effective viscosity can be prescribed by our closed-form expressions for both translational and rotational cases. Next, the polymer depletion effect and the Stokes theory are briefly reviewed, followed by our derivation of correction functions based on the two-layer approximation.

\section{THEORY}

In Sec. II A, we review the equilibrium density and the corresponding viscosity profiles resulting from polymer depletion around a spherical particle. Stokes's stream function theory is summarized in Sec. II B using dimensionless quantities. The simplified two-layer approximation is presented in Sec. II C. The final result is formulated in terms of an analytical correction function to Stokes' law. The apparent slip length is also analytically quantified. To make the presentation clear, unnecessary symbols for the dimensionless parameters are removed to simplify the notation.

\section{A. Polymer density and viscosity profile near a nonadsorbing surface}

The viscosity profile, determined by the polymer segment density, is the physical origin of the apparent slip affecting the transport phenomena of a spherical particle that moves through a polymer solution (see Fig. 1). Near a nonadsorbing planar surface, the local polymer concentration $c$ is a function of the distance $z$ away from the wall position. $c \rightarrow 0$ as $z \rightarrow 0$, and $c$ approaches the bulk value $c_{b}$ as $z \rightarrow \infty$. As a first approximation $[26,27]$ one might simplify the density profile

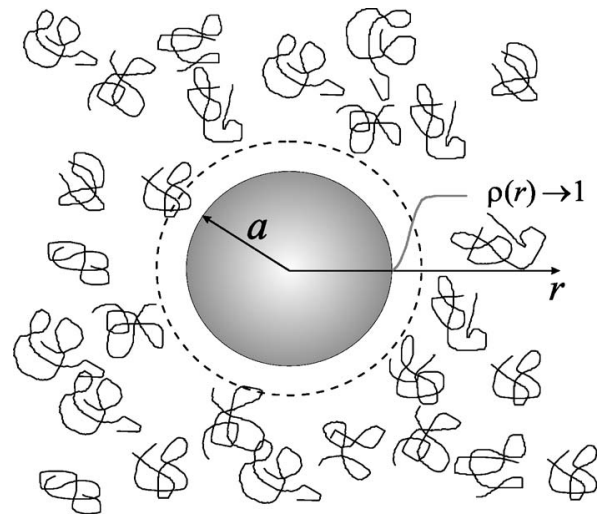

FIG. 1. Schematic of a sphere in a polymer solution containing nonadsorbing chains, and the normalized density profile.

in the region near the flat wall using a unit step function,

$$
\rho(z)=\left\{\begin{array}{l}
0 \text { for } 0 \leqslant z \leqslant \delta_{w}, \\
1 \text { for } z>\delta_{w},
\end{array}\right.
$$

where $\rho(z)=c(z) / c_{b}$ is the normalized segment density and $\delta_{w}$ is the depletion thickness at the wall. In reality the density profile varies gradually in the region near a flat wall. An accurate density profile

$$
\rho(z)=\tanh ^{2}\left(z / \delta_{w}\right)
$$

was first derived by de Gennes [29] for a semidilute polymer solution near a planar wall as a mean-field approximation. In that case $\delta_{w}$ equals the mean-field correlation length. In a dilute polymer solution, $\delta_{w}$ is very close to the polymer's radius of gyration $R_{g}[30,37]$. Equation (2) holds, however, quite generally [31] for ideal chains $[30,38]$, chains in the excluded volume limit [39], and mean-field chains for arbitrary concentration [37]. The depletion thickness $\delta_{w}$ is related to the negative adsorbed amount of polymer chains $\Gamma$ by $\delta_{w}=\Gamma / c_{b}$ [37] and follows the definition of the zeroth moment of the excess $[1-\rho(z)][37,38]$

$$
\delta_{w}=\int_{0}^{\infty}[1-\rho(z)] d z
$$

which is consistent with the formulation in Eq. (2). Similarly, the density profile around a spherical particle can be simplified by the Asakura-Oosawa-Vrij formulation [25-27]

$$
\rho(r)=\left\{\begin{array}{l}
0 \text { for } 1 \leqslant r \leqslant 1+\delta_{s}, \\
1 \text { for } r>1+\delta_{s},
\end{array}\right.
$$

where $\delta_{s}$ is the depletion thickness around a sphere, $\rho(r)$ $=c(r) / c_{b}$ is the normalized local density, and $r$ is the radial distance away from the center point of the spherical particle. For relatively large spheres in a dilute polymer solution, $\delta_{s}$ $\simeq R_{g}$. Note that all distance-related parameters including $r, z$, $\delta_{w}$, and $\delta_{s}$ are scaled by the particle radius $a$ as the characteristic length here and in the following sections except those expressions specifically notified for demonstrating the results. The viscosity profile relates to the density profile [31] as $\eta(r)=\eta_{s}\left[1+[\eta] c_{b} \rho(r)+k_{H}\left([\eta] c_{b} \rho(r)\right)^{2}+\cdots\right]$ where $\eta(r)$ is 
the local viscosity of the polymer solution, $\eta_{s}$ is the solvent viscosity, $[\eta]$ is the intrinsic viscosity, and $k_{H}$ is the Huggins coefficient. The profile is identical to the Huggins equation for $\rho=1$. Here we apply a step function for the viscosity corresponding to Eq. (4), i.e.,

$$
\eta(r)= \begin{cases}\eta_{s} & \text { for } 1 \leqslant r \leqslant 1+\delta_{s}, \\ \eta_{p} & \text { for } r>1+\delta_{s} .\end{cases}
$$

We thus assume the polymer relaxation time is short to such a degree that the polymer solution including depletion layer surrounding the sphere does not deform while the sphere is moving. Ignoring these relaxation effects means that the time relaxations of the chains are much faster than the relaxation time scale of the sphere.

\section{B. Stokes' law and non-Stokes-Einsteinian behavior}

In a homogeneous medium, the velocity and pressure fields satisfy the following momentum and continuity equations based on Stokes' approximation [40-42],

$$
\boldsymbol{\nabla} p=\boldsymbol{\nabla}^{2} \mathbf{v}, \quad \boldsymbol{\nabla} \cdot \mathbf{v}=0 .
$$

The system is nondimensionalized by scaling the length, velocity vector $\mathbf{v}$, and pressure $p$ by the particle radius $a$, the magnitude of the translational velocity $\mathbf{U}=U \hat{\mathbf{e}}_{z}$, and the shear stress $\eta U / a$, respectively, where $\eta$ is the constant dynamic viscosity. For translational motion the momentum equation is simplified by using the Stokes stream function $\psi(r, \theta)[43]$ in terms of spherical polar coordinates where $\theta$ denotes the polar coordinate and the system is symmetric in the azimuthal direction. The stream function $\psi(r, \theta)$, scaled by $U a^{2}$, is related to the velocity field $\mathbf{v}=v_{r} \hat{\mathbf{e}}_{r}+v_{\theta} \hat{\mathbf{e}}_{\theta}$ by $v_{r}$ $=-\left(r^{2} \sin \theta\right)^{-1} \partial \psi / \partial \theta$ and $v_{\theta}=(r \sin \theta)^{-1} \partial \psi / \partial r$. Mediated by the vorticity equation, the momentum equation can be simplified to a separable fourth-order differential equation for $\psi$. Complemented by the vanishing far-field and no-slip boundary conditions, the stream function and the corresponding velocity and pressure fields can be found. Note that the normal stress $\sigma_{r r}$ vanishes at the particle surface. The total drag force $F$ is calculated by the rate of viscous dissipation over the entire flow induced by the particle translation, or by the area integration of the pressure and shear stress over the particle surface. The dimensionless result of Stokes' law states $\mathbf{F}=-6 \pi \hat{\mathbf{e}}_{z}$, where the total drag force $\mathbf{F}$ is scaled by $\eta a U$, and the negative sign shows that the force is opposite to the moving direction of the particle.

For a rotational motion in a quiescent fluid, the shear flow is driven by a rotating sphere with angular velocity $\boldsymbol{\Omega}=\Omega \hat{\mathbf{e}}_{z}$, where $\hat{\mathbf{e}}_{z}$ indicates the rotating axis. The velocity at the surface of the sphere is $\boldsymbol{\Omega} \times \mathbf{r}(a, \theta, \varphi)$, where $\mathbf{r}(r, \theta, \varphi)$ is the position vector in spherical polar coordinates. The system parameters are independent of $\varphi$, implying that the shearinduced velocity field can be found by using a trial solution $\mathbf{v}=\left(0,0, v_{\varphi}\right)=(\boldsymbol{\Omega} \times \mathbf{r}) \widetilde{w}(r)$ with vanishing velocity components in both $r$ and $\theta$ directions. The trial solution satisfies the continuity equation, and according to the Stokes equation in $r$ and $\theta$ directions, the pressure field is a constant. Applying $\mathbf{v}=v_{\varphi} \hat{\mathbf{e}}_{\varphi}$ where $v_{\varphi}=\Omega \sin (\theta) w(r)$, the momentum equation can be simplified to

$$
\left[\nabla^{2} \mathbf{v}\right]_{\varphi}=\nabla^{2} v_{\varphi}-\frac{v_{\varphi}}{r^{2} \sin ^{2} \theta}=0 .
$$

We further nondimensionalize the system by the following scales: Length $\sim a$, velocity $\mathbf{v} \sim \Omega a$, stress tensor $\boldsymbol{\tau} \sim \eta \Omega$, and the torque acting on the sphere $\mathbf{T} \sim \eta \Omega a^{3}$. The induced velocity field can be solved by substituting the dimensionless trial solution $v_{\varphi}=\sin (\theta) w(r)$ into Eq. (7) and applying the vanishing and no-slip boundary conditions. The final result for the shear-induced torque on the sphere is given by

$$
\mathbf{T}=\int_{\partial \Omega} \mathbf{r} \times(\boldsymbol{\tau} \cdot \mathbf{n}) d A=-8 \pi \hat{\mathbf{e}}_{z},
$$

where $\mathbf{n}$ is the surface normal and the negative sign shows that the resistance is opposite to the rotational direction.

In terms of the friction coefficient, $\mathbf{F}=-f^{t} \mathbf{U}$ and $\mathbf{T}$ $=-f^{r} \boldsymbol{\Omega}$. Thus, in a complex fluid, Stokes' calculation gives $f_{\mathrm{eff}}^{t}=6 \pi \eta_{\mathrm{eff}}^{t} a$ and $f_{\mathrm{eff}}^{r}=8 \pi \eta_{\mathrm{eff}}^{r} a^{3}$, where $\eta_{\mathrm{eff}}=\eta_{s}$ in the case of a sphere translating or rotating in a pure solvent with viscosity $\eta_{s}$, and one may ad hoc postpone $\eta_{\mathrm{eff}}=\eta_{p}$ in the case of a polymer solution with viscosity $\eta_{p}$. Dynamic light scattering measurements of the diffusion behaviors of colloidal spheres in a polymer solution yield translational and rotational diffusion coefficients $D^{t}$ and $D^{r}$, respectively. In a dilute or semidilute system, the thermal energy $k_{B} T$ and apparent friction a sphere experiences are connected to the effective diffusivities through the Einstein relation $D_{\text {eff }}^{t}$ $=k_{B} T / f_{\text {eff }}^{t}$ for translation motion and $D_{\text {eff }}^{r}=k_{B} T / f_{\text {eff }}^{r}$ for rotational motion. Therefore, the corresponding Stokes-Einstein and Stokes-Einstein-Debye relations are written as

$$
D_{\mathrm{eff}}^{t}=\frac{k_{B} T}{6 \pi \eta_{\mathrm{eff}}^{t} a}, \quad D_{\mathrm{eff}}^{r}=\frac{k_{B} T}{8 \pi \eta_{\mathrm{eff}}^{r} a^{3}} .
$$

Measurements have shown that $\eta_{\text {eff }}$ depends on the ratio of $a$ to $\xi$, where $\xi$ is the typical length scale in the polymer solution $[2-7,16]$. The case $\eta_{\text {eff }}=\eta_{s}$ seems to hold in the limit $a \ll O(\xi)$. In dilute polymer solutions the length scale would be the polymer's radius of gyration and, in concentrated polymer solution, it equals the correlation length which decreases as the polymer concentration increases above the coil overlap concentration. However, the finding of Eq. (9) from experimental data is in contrast to the result proposed by Donath et al. [6] who stated the friction coefficient for translational motion is $4 \pi \eta_{p} a$ for the case of small $a$. In their analysis, the friction coefficient $4 \pi \eta_{p} a$, which corresponds to the full slip limit, is not justified because the no-slip boundary condition is still valid for the solvent molecules. In the opposite limit $a \gg O(\xi)$ experimental results indicate that $\eta_{\text {eff }} \rightarrow \eta_{p}$. Thus in the transition regime with $a \sim O(\xi)$ one needs to evaluate $D_{\text {eff }}^{t}$ and $D_{\text {eff }}^{r}$ by establishing an effective viscosity $\eta_{\text {eff }}$, which lies in between the limiting values; that is, $\eta_{s}<\eta_{\text {eff }}<\eta_{p}$. The corresponding diffusive behavior is often termed non-Stokes-Einsteinian.

\section{Two-layer approximation}

Following Stokes' stream function theory, we derive here the analytical expressions of the correction functions $g^{t}$ and 


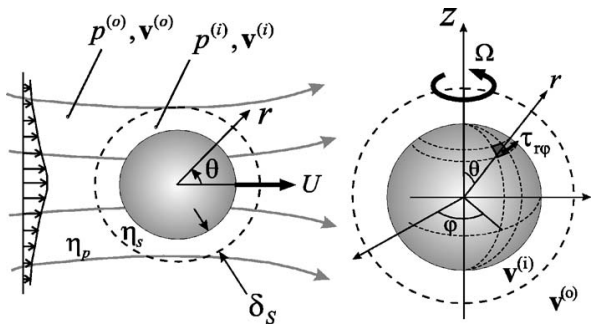

FIG. 2. Creeping flow induced by the translational (left) and rotational (right) motion of a sphere in a solution with polymer depletion effect. The sketch illustrates the streamlines, induced flow, coordinate system, and the physical domains for the two-layer approximation.

$g^{r}$, defined by $\eta_{\mathrm{eff}}^{t}=\eta_{s} g^{t}$ and $\eta_{\mathrm{eff}}^{r}=\eta_{s} g^{r}$, for translational and rotational motions, respectively (Fig. 2).

\section{Translational motion}

The simplified viscosity profile is described as a step function corresponding to the polymer segment density profile given in Eq. (4). The fluid dynamics in the depleted inner and unaffected outer layers can be written as

$$
\boldsymbol{\nabla} p^{(i)}=\nabla^{2} \mathbf{v}^{(i)}, \quad \boldsymbol{\nabla} \cdot \mathbf{v}^{(i)}=0 \text { for } 1 \leqslant r \leqslant 1+\delta_{s},
$$

$$
\lambda \boldsymbol{\nabla} p^{(o)}=\nabla^{2} \mathbf{v}^{(o)}, \quad \boldsymbol{\nabla} \cdot \mathbf{v}^{(o)}=0 \quad \text { for } 1+\delta_{s} \leqslant r<\infty,
$$

where $\delta_{s}$ is the dimensionless thickness of the depletion layer around the sphere, and $\lambda=\eta_{s} / \eta_{p}$ is the relative viscosity between the inner and outer layers. Note that $\eta_{s}$ and $\eta_{p}$ are fixed viscosities corresponding to the densities $\rho=0$ and $\rho$ $=1$; thus $\eta_{s} \leqslant \eta_{p}$ and $\lambda \leqslant 1$. The governing equations are nondimensionalized by scaling the length, velocity vector $\mathbf{v}$, the stress tensor $\tau_{i j}$, and thus the pressure field $p$ through

$$
\text { length } \sim a, \quad \mathbf{v} \sim U, \quad \tau_{i j} \text { and } p \sim \eta_{s} U / a,
$$

where $\tau_{i j}=-p \delta_{i j}+\sigma_{i j}$ is the total stress tensor, $\delta_{i j}$ is the Kronecker delta, and $\sigma_{i j}$ is the viscous stress tensor. Applying Stokes stream function to the system leads to

$$
\begin{aligned}
& E^{4} \psi^{(i)}=0 \quad \text { for } 1 \leqslant r \leqslant 1+\delta_{s}, \\
& E^{4} \psi^{(o)}=0 \quad \text { for } 1+\delta_{s} \leqslant r<\infty,
\end{aligned}
$$

where

$$
E^{4} \equiv\left[\frac{\partial^{2}}{\partial r^{2}}+\frac{\sin \theta}{r^{2}} \frac{\partial}{\partial \theta}\left(\frac{1}{\sin \theta} \frac{\partial}{\partial \theta}\right)\right]^{2}
$$

The inner-layer stream function $\psi^{(i)}(r, \theta)$ and the outer-layer stream function $\psi^{(o)}(r, \theta)$ are related to the corresponding velocity fields by

$$
v_{r}^{(i)}=\frac{-1}{r^{2} \sin \theta} \frac{\partial \psi^{(i)}}{\partial \theta}, \quad v_{\theta}^{(i)}=\frac{1}{r \sin \theta} \frac{\partial \psi^{(i)}}{\partial r},
$$

$$
v_{r}^{(o)}=\frac{-1}{r^{2} \sin \theta} \frac{\partial \psi^{(o)}}{\partial \theta}, \quad v_{\theta}^{(o)}=\frac{1}{r \sin \theta} \frac{\partial \psi^{(o)}}{\partial r} .
$$

Substituting trial solutions, $\psi^{(i)}(r, \theta)=\sin ^{2}(\theta) f^{(i)}(r)$ and $\psi^{(o)}(r, \theta)=\sin ^{2}(\theta) f^{(o)}(r)$, into Eqs. (12a) and (12b), the resulting equations for the unknown functions $f^{(i)}$ and $f^{(o)}$ can be written as

$$
\begin{gathered}
f^{(i)(i v)}-\frac{4 f^{(i)^{\prime \prime}}}{r^{2}}+\frac{8 f^{(i)^{\prime}}}{r^{3}}-\frac{8 f^{(i)}}{r^{4}}=0 \text { for } 1 \leqslant r \leqslant 1+\delta_{s}, \\
f^{(o)(i v)}-\frac{4 f^{(o)^{\prime \prime}}}{r^{2}}+\frac{8 f^{(o)^{\prime}}}{r^{3}}-\frac{8 f^{(o)}}{r^{4}}=0 \text { for } 1+\delta_{s} \leqslant r<\infty,
\end{gathered}
$$

of which the general solutions are

$$
\begin{aligned}
& f^{(i)}(r)=A r^{4}+B r^{2}+C r+\frac{D}{r}, \\
& f^{(o)}(r)=E r^{4}+F r^{2}+G r+\frac{H}{r},
\end{aligned}
$$

respectively. The system is complemented by the following boundary conditions:

$$
\begin{gathered}
v_{r}^{(o)}, v_{\theta}^{(o)} \rightarrow 0 \quad \text { as } \quad r \rightarrow \infty, \\
\sigma_{r \theta}^{(i)}=\sigma_{r \theta}^{(o)} \quad \text { at } \quad r=1+\delta_{s}, \\
-p^{(i)}+\sigma_{r r}^{(i)}=-p^{(o)}+\sigma_{r r}^{(o)} \quad \text { at } \quad r=1+\delta_{s}, \\
v_{r}^{(i)}=v_{r}^{(o)} \quad \text { at } \quad r=1+\delta_{s}, \\
v_{\theta}^{(i)}=v_{\theta}^{(o)} \quad \text { at } \quad r=1+\delta_{s}, \\
v_{r}^{(i)}=\cos \theta \quad \text { at } \quad r=1, \\
v_{\theta}^{(i)}=-\sin \theta \quad \text { at } \quad r=1 .
\end{gathered}
$$

The fluid dynamics in the inner and outer layers are coupled through boundary conditions at $r=1+\delta_{s}$. The seven boundary conditions are sufficient to determine the eight coefficients $A \sim H$. The vanishing velocity boundary condition in the far field, Eq. (17a), requires $E=0$ and $F=0$. Equations (17b) and (17c) impose the continuity of the shear and total normal stress fields across the outer boundary of the depletion layer or the inner boundary of the bulk polymer solution. Equations (17d) and (17e) match the velocity field and ensure inner and outer stream functions are continuous across the boundary at $r=1+\delta_{s}$. Equations $(17 \mathrm{f})$ and $(17 \mathrm{~g})$ are noslip conditions. In terms of spherical coordinates, Eq. (17b) can be expressed as

$$
\lambda\left[r \frac{\partial}{\partial r}\left(\frac{v_{\theta}^{(i)}}{r}\right)+\frac{1}{r} \frac{\partial v_{r}^{(i)}}{\partial \theta}\right]=\left[r \frac{\partial}{\partial r}\left(\frac{v_{\theta}^{(o)}}{r}\right)+\frac{1}{r} \frac{\partial v_{r}^{(o)}}{\partial \theta}\right] .
$$




$$
\lambda\left(f^{(i)^{\prime \prime}}-\frac{2 f^{(i)^{\prime}}}{r}+\frac{2 f^{(i)}}{r^{2}}\right)=f^{(o)^{\prime \prime}}-\frac{2 f^{(o)^{\prime}}}{r}+\frac{2 f^{(o)}}{r^{2}},
$$

which gives the algebraic relation

$$
\lambda\left(1+\delta_{s}\right)^{5} A+\lambda D-H=0 .
$$

Using Stokes' stream functions and integrating the momentum equations Eqs. (10a) and (10b), the pressure fields $p^{(i)}$ and $p^{(o)}$ can be formulated in terms of the undetermined coefficients as

$$
p^{(i)}\left(1+\delta_{s}, \theta\right)=-20\left(1+\delta_{s}\right) \cos \theta A-\frac{2 \cos \theta}{\left(1+\delta_{s}\right)^{2}} C
$$

and

$$
p^{(o)}\left(1+\delta_{s}, \theta\right)=\frac{-2 \cos \theta}{\lambda\left(1+\delta_{s}\right)^{2}} G
$$

provided the reference pressure is set to zero at infinity. Thus Eq. (17c) reduces to

$$
2\left(1+\delta_{s}\right)^{5} A+\left(1+\delta_{s}\right)^{2} C+2 D-\frac{\left(1+\delta_{s}\right)^{2}}{\lambda} G-\frac{2}{\lambda} H=0 .
$$

Boundary conditions, Eqs. (17d) $-(17 \mathrm{~g})$ yield the following relations:

$$
\left(1+\delta_{s}\right)^{5} A+\left(1+\delta_{s}\right)^{3} B+\left(1+\delta_{s}\right)^{2} C+D-\left(1+\delta_{s}\right)^{2} G-H=0,
$$

$$
\begin{gathered}
4\left(1+\delta_{s}\right)^{5} A+2\left(1+\delta_{s}\right)^{3} B+\left(1+\delta_{s}\right)^{2} C-D \\
-\left(1+\delta_{s}\right)^{2} G+H=0, \\
A+B+C+D=-1 / 2, \\
4 A+2 B+C-D=-1,
\end{gathered}
$$

respectively. Solving the algebraic system formed by Eqs. (20) and (23), and Eqs. (24)-(27) leads to the exact solution:

$$
\begin{gathered}
A=\frac{3}{2}(1-\lambda)\left(1+\delta_{s}\right)\left[1-\left(1+\delta_{s}\right)^{2}\right] / \Pi, \\
B=\frac{-1}{2}\left[-3(3+2 \lambda)(1-\lambda)\left(1+\delta_{s}\right)^{5}\right. \\
\left.+5(1-\lambda)\left(1+\delta_{s}\right)^{3}+4(1-\lambda)^{2}\right] / \Pi, \\
C=\frac{-3}{2}\left(1+\delta_{s}\right)\left[(2+3 \lambda)\left(1+\delta_{s}\right)^{5}-2(1-\lambda)\right] / \Pi, \\
D=\frac{1}{2}\left(1+\delta_{s}\right)^{3}\left[(2+3 \lambda)\left(1+\delta_{s}\right)^{3}-2(1-\lambda)\right] / \Pi, \\
G=\frac{-3}{2} \lambda\left(1+\delta_{s}\right)\left[(2+3 \lambda)\left(1+\delta_{s}\right)^{5}-2(1-\lambda)\right] / \Pi,
\end{gathered}
$$

$$
\begin{aligned}
H= & \frac{1}{2} \lambda\left(1+\delta_{s}\right)^{3}\left[-3(1-\lambda)\left(1+\delta_{s}\right)^{5}\right. \\
& \left.+5\left(1+\delta_{s}\right)^{3}-2(1-\lambda)\right] / \Pi,
\end{aligned}
$$

where

$$
\begin{aligned}
\Pi= & 2(2+3 \lambda)\left(1+\delta_{s}\right)^{6}-3(3+2 \lambda)(1-\lambda)\left(1+\delta_{s}\right)^{5} \\
& +10(1-\lambda)\left(1+\delta_{s}\right)^{3}-9(1-\lambda)\left(1+\delta_{s}\right)+4(1-\lambda)^{2}
\end{aligned}
$$

for $0 \leqslant \lambda \leqslant 1$. Note that this result may be extended to the case of polymer adsorption for parameters $\lambda \geqslant 1$ with properly defined adsorbed layer thickness. In summary, the stream functions solved for the two-layer approximation are $\psi^{(i)}=\left(A r^{4}+B r^{2}+C r+D / r\right) \sin ^{2} \theta$ and $\psi^{(o)}=(G r+H / r) \sin ^{2} \theta$ where the constants $A \sim H$ are functions of $\lambda$ and $\delta_{s}$, given by Eq. (28). Thus the velocity, pressure, normal, and shear stress fields can be established accordingly. The total drag force is determined by integrating the surface traction. Following the stress field obtained, the modified Stokes law can be expressed as $\mathbf{F}=-6 \pi g^{t} \hat{\mathbf{e}}_{z}$, where $\mathbf{F}$ is scaled by $\eta_{s} a U$ and the correction function for the translation motion $g^{t}$ is

$$
g^{t}\left(\lambda, \delta_{s}\right)=\frac{1}{\Pi}\left[2(2+3 \lambda)\left(1+\delta_{s}\right)^{6}-4(1-\lambda)\left(1+\delta_{s}\right)\right] .
$$

The denominator $\Pi$ is defined in Eq. (28). This result represents the effective viscosity or an equivalent friction constant in a closed form, incorporating the polymer depletion effect for the translational motion. A few limiting cases thus can be recognized: (a) When $\lambda=1, g^{t}=1$, the resistance follows Stokes' law with an effective viscosity that equals the solvent viscosity; (b) for a very thick depletion layer, $\delta_{s} \gg O(1)$, the sixth-order terms dominate, and thus $g^{t} \rightarrow 1$; (c) for a very thin depletion layer with $\delta_{s} \ll \lambda \leqslant 1$,

$$
g^{t} \sim \frac{1}{\lambda}-\frac{(1-\lambda)}{\lambda^{2}} \delta_{s}+\frac{3(1-\lambda)}{\lambda^{3}} \delta_{s}^{2} .
$$

We thus recover the well-known limiting behaviors encountered experimentally [7], that is, if the depletion layer is very large compared to the sphere radius, we have $g^{t} \rightarrow 1$ or $\eta_{\text {eff }} \rightarrow \eta_{s}$ as described in case (b). In the case of a small depletion layer compared to the sphere radius, $g^{t}$ is corrected from the viscosity of the polymer solution by small terms resolved in Eq. (30). Thus for a very thin depletion layer, when $\eta_{s} \rightarrow \eta_{p}$ or $\lambda \rightarrow 1, g^{t} \sim \lambda^{-1}$ is a good approximation for the effective viscosity, which is equal to the viscosity of the polymer solution in the outer layer. Qualitatively, these results are comparable to the theoretical prediction for translational motion of spheres through networks of hard rods $[19,20]$, using a different approach where direct and hydrodynamic interactions between the sphere and the rods are explicitly accounted for.

\section{Rotational motion}

Following Eq. (7) the governing system for the rotational motion can be written as 


$$
\begin{aligned}
& \nabla^{2} v_{\varphi}^{(i)}-\frac{v_{\varphi}^{(i)}}{r^{2} \sin ^{2}(\theta)}=0 \text { for } 1 \leqslant r \leqslant 1+\delta_{s}, \\
& \nabla^{2} v_{\varphi}^{(o)}-\frac{v_{\varphi}^{(o)}}{r^{2} \sin ^{2}(\theta)}=0 \text { for } 1+\delta_{s} \leqslant r<\infty .
\end{aligned}
$$

The characteristic scales are defined by

$$
\text { length } \sim a, \quad \mathbf{v} \sim \Omega a^{\prime}, \quad \boldsymbol{\tau} \sim \eta_{s} \Omega, \quad \mathbf{T} \sim \eta_{s} \Omega a^{3} .
$$

Substituting trial solutions $v_{\varphi}^{(i)}(r, \theta)=\sin (\theta) w^{(i)}(r)$ and $v_{\varphi}^{(o)}(r, \theta)=\sin (\theta) w^{(o)}(r)$ into Eqs. (31a) and (31b), the resulting equations for the unknown functions $w^{(i)}$ and $w^{(o)}$ become

$$
\begin{gathered}
w^{(i)^{\prime \prime}}+\frac{2}{r} w^{(i)^{\prime}}-\frac{2}{r^{2}} w^{(i)}=0 \quad \text { for } 1 \leqslant r \leqslant 1+\delta_{s}, \\
w^{(o)^{\prime \prime}}+\frac{2}{r} w^{(o)}-\frac{2}{r^{2}} w^{(o)^{\prime}}=0 \quad \text { for } 1+\delta_{s} \leqslant r<\infty .
\end{gathered}
$$

The corresponding general solutions of Eqs. (33a) and (33b) are $w^{(i)}=M / r^{2}+N r$ and $w^{(o)}=I / r^{2}+J r$, respectively. The system is subjected to four boundary conditions:

$$
\begin{gathered}
v_{\varphi}^{(o)} \rightarrow 0 \quad \text { as } r \rightarrow \infty, \\
v_{\varphi}^{(i)}=\sin \theta \quad \text { at } r=1, \\
v_{\varphi}^{(i)}=v_{\varphi}^{(o)} \quad \text { at } r=1+\delta_{s}, \\
\sigma_{r \varphi}^{(i)}=\sigma_{r \varphi}^{(o)} \quad \text { at } r=1+\delta_{s} .
\end{gathered}
$$

Solving for the constants $M, N, I$, and $J$ using Eqs. (34a)-(34d) leads to the velocity and stress fields. The torque acting on the sphere is evaluated by the area integration $\int_{\partial \Omega} \sin (\theta) \tau_{r \varphi}^{(i)} d A$ at $r=1$. Finally, we obtain the solution for the torque $\mathbf{T}=-8 \pi g^{r} \hat{\mathbf{e}}_{z}$ in terms of the correction function

$$
g^{r}\left(\lambda, \delta_{s}\right)=\frac{\left(1+\delta_{s}\right)^{3}}{\left(1+\delta_{s}\right)^{3}+\lambda-1} .
$$

A few limiting cases for $g^{r}$ can also be recognized: (a) $\lambda$ $=1, g^{r}=1$, the effective viscosity equals the solvent viscosity; (b) for a thick depletion layer, $\delta_{s} \gg O(1)$, we have $g^{r} \rightarrow 1$; (c) for a very thin depletion layer with $\delta_{s} \ll \lambda \leqslant 1$,

$$
g^{r} \sim \frac{1}{\lambda}-\frac{3(1-\lambda)}{\lambda^{2}} \delta_{s}+\frac{9-12 \lambda+3 \lambda^{2}}{\lambda^{3}} \delta_{s}^{2} .
$$

Similar to the translational case, if the depletion layer is very large compared to the sphere radius, we have $g^{r} \rightarrow 1$ or $\eta_{\text {eff }}$ $\rightarrow \eta_{s}$ as described in case (b). In the case of a small depletion layer compared to the sphere radius, $g_{r}$ is corrected from the viscosity of the polymer solution by small terms including $\delta_{s}$, as resolved in Eq. (36). Note that for cases with a thin depletion layer, the asymptote Eq. (36) is valid only if $\lambda$ is not too small. However, Eq. (35) holds for $0 \leqslant \lambda \leqslant 1$ al-

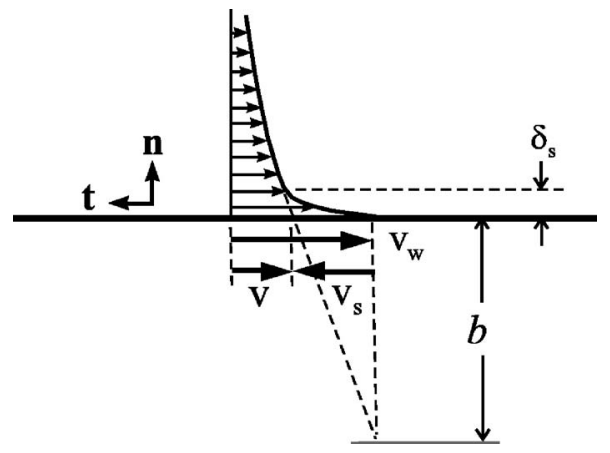

FIG. 3. Schematic of tangential velocity field induced by a moving boundary and the apparent slip that represents the polymer depletion effect. Linear extrapolation of the velocity profile of the outer layer gives the slip length $b$.

though $\eta_{s}=0$ or $f^{r}=0$ is beyond the scope of a practical system.

\section{Apparent slip effect}

An alternative way to characterize the effect of polymer depletion on sphere motion is by introducing an equivalent slip effect at the solid-liquid interface. The Navier boundary condition assumes that the slippery fluid element immediately in contact with the solid surface is resisted by the tangential traction $f_{t}$, which is proportional to the slip (tangential) velocity $v_{s}[40,44]$. The proportional constant is defined as the friction coefficient $\beta$ by letting

$$
f_{t}=\beta v_{s}
$$

If the fluid flow with velocity field $\mathbf{v}$ is driven by the moving boundary with wall velocity $\mathbf{v}_{w}$, the kinematic boundary conditions that incorporate the slip effect can be formulated by

$$
\begin{aligned}
& \left(\mathbf{v}-\mathbf{v}_{w}\right) \cdot \mathbf{n}=0, \\
& \left(\mathbf{v}-\mathbf{v}_{w}\right) \cdot \mathbf{t}=v_{s},
\end{aligned}
$$

where the surface normal $\mathbf{n}$ and surface tangent $\mathbf{t}$ represent the local coordinates along the solid boundary (Fig. 3). The slip velocity $v_{s}$ only appears along the tangential direction $\mathbf{t}$. Resolving a particle-induced flow including slip effect requires the vanishing far-field condition and the kinematic boundary conditions, Eq. (38a), and the combination of Eqs. (37) and (38b),

$$
\beta\left(v_{\theta}+\sin \theta\right)=\sigma_{r \theta} \quad \text { at } r=1 .
$$

The following stream function can be derived

$$
\psi(r, \theta)=\left[\frac{\beta}{4(\beta+3) r}-\frac{3(\beta+2) r}{4(\beta+3)}\right] \sin ^{2} \theta,
$$

and, consequently, the velocity, pressure, normal, and shear stress fields can be found. The total force acting on a slippery sphere was first derived by Basset [45], expressed as 


$$
\mathbf{F}_{\text {slip }}=-6 \pi \frac{\beta+2}{\beta+3} \hat{\mathbf{e}}_{z},
$$

where the total drag force $\mathbf{F}_{\text {slip }}$ is scaled by $\eta_{p} a U$ using a uniform viscosity of the polymer solution $\eta_{p}$, and the friction coefficient $\beta$ is scaled by $\eta_{p} / a$. When the no-slip condition applies, $\beta \rightarrow \infty$ and Eq. (41) reproduces Stokes' law; when the perfect slip applies, $\beta \rightarrow 0$ and the total resistance is reduced by a factor of $2 / 3$ [41]. Similarly, the modified boundary condition for a rotating sphere with slippery surface satisfies

$$
\beta\left(v_{\varphi}-\sin \theta\right)=\sigma_{r \varphi} \quad \text { at } r=1 .
$$

We thus obtain

$$
v_{\varphi}=\frac{\sin \theta}{r^{2}}\left(\frac{\beta}{\beta+3}\right), \quad \sigma_{r \varphi}=-\frac{3 \sin \theta}{r^{3}}\left(\frac{\beta}{\beta+3}\right),
$$

and the torque acting on the sphere is

$$
\mathbf{T}_{\text {slip }}=-8 \pi\left(\frac{\beta}{\beta+3}\right) \hat{\mathbf{e}}_{z},
$$

where $\mathbf{T}_{\text {slip }}$ is scaled by $\eta_{p} \Omega a^{3}$. When the no-slip condition applies, $\beta \rightarrow \infty$ and Eq. (44) reproduces Stokes' law; when the perfect slip applies, $\beta \rightarrow 0$, and the total resistance vanishes for the rotating sphere. It is also convenient to define the slip boundary condition in terms of an apparent slip length $b$ from linear extrapolation of the velocity profile [31]. In dimensionless form, $b=1 / \beta$, and $b$ is scaled by the radius $a$ of the sphere. Finally, the two-layer approximation can be formulated in terms of the apparent slip effect for both translational and rotational motions based on the correction factors. Comparing the correction factors gives $\left(\beta^{t}+2\right) /\left(\beta^{t}+3\right)$ $=\lambda g^{t}$ where $\beta^{t}=1 / b^{t}$. Thus for the cases with thin polymer depletion layer, the apparent slip length for the translational motion is approximated by

$$
b^{t} \sim \frac{(1-\lambda)}{\lambda} \delta_{s}-\frac{3(1-\lambda)}{\lambda} \delta_{s}^{2} .
$$

Similarly, $\beta^{r} /\left(\beta^{r}+3\right)=\lambda g^{r}$, where $\beta^{r}=1 / b^{r}$, and

$$
b^{r} \sim \frac{(1-\lambda)}{\lambda} \delta_{s}-\frac{2(1-\lambda)}{\lambda} \delta_{s}^{2}
$$

estimates the apparent slip length for the rotation motion. Both $b^{t}$ and $b^{r}$, having consistent magnitude to the first-order approximation, are functions of $\lambda$ and $\delta_{s}$. The no-slip condition corresponds to $\lambda=1$, and the slip length increases as $\lambda$ reduces. A perfect slip condition is achieved as $\lambda \ll \delta_{s} \ll 1$, in which $b^{t}, b^{r} \rightarrow \infty$ and $\beta^{t}, \beta^{r} \rightarrow 0$. As a final test for our exact solution, by letting $\delta_{s}=10^{-3}$ and $\lambda=10^{-5}, b^{t}$ and $b^{r}$ approach $100, \beta^{t}$ and $\beta^{r}$ approach 0.01, $\mathbf{T}_{\text {slip }}$ vanishes, and $\lambda g^{t} \rightarrow 2 / 3$. This limit is consistent with the perfect slip condition given by Eq. (41) for the translational motion.

\section{RESULTS AND DISCUSSION}

In this section we illustrate the theoretical results for the modified transport properties of a sphere through a polymer

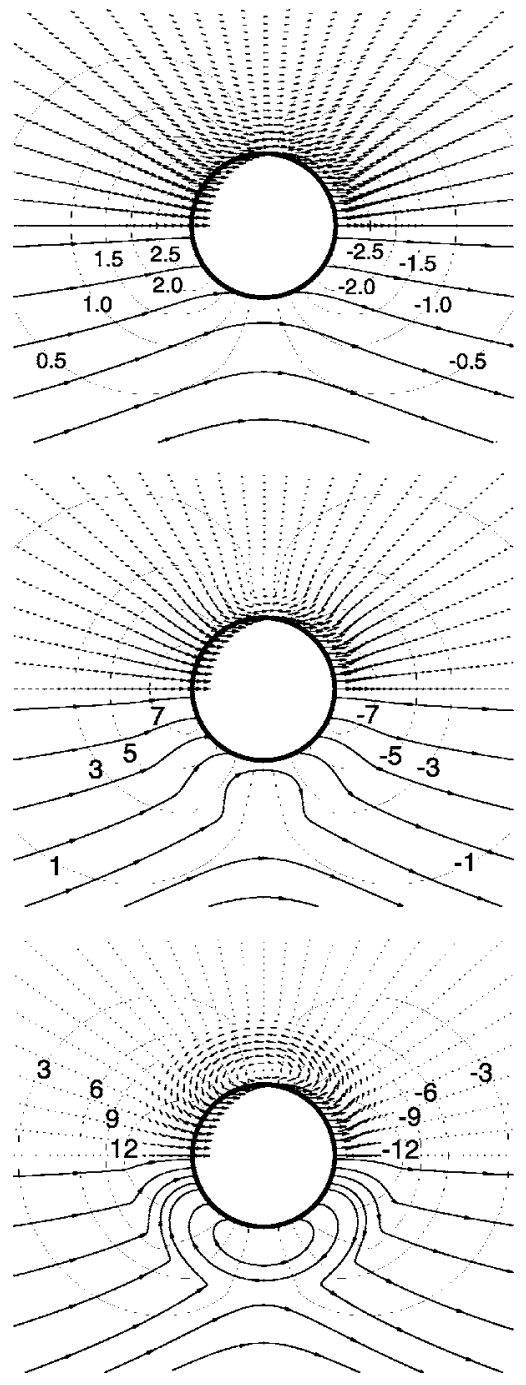

FIG. 4. Velocity vectors, streamlines, and contours for the total normal stress field based on the two-layer model for a sphere translating to the right. The stress field is normalized by $\eta_{s} U / a$. Parameters applied: $\delta_{s}=1$ for all; $\lambda=0.5$ (top); $\lambda=0.1$ (middle); $\lambda=0.01$ (bottom).

solution. The flow patterns driven by the particle's translational motion are illustrated in Fig. 4. When the viscosity ratio $\lambda$ is sufficiently small and the depletion thickness is of the order of the particle radius, the circulation induced by the strong total normal stress can be clearly seen. The front and aft symmetric pattern indicates the reversibility of Stokes' flow.

Figure 5 shows the correction function $g^{t}$, formulated by Eq. (29), versus the specific viscosity $\eta_{\mathrm{sp}}$ of the bulk polymer solution. The specific viscosity, defined by $\eta_{\mathrm{sp}}=\left(\eta_{p}\right.$ $\left.-\eta_{s}\right) / \eta_{s}=\lambda^{-1}-1$, characterizes the increase of viscosity by increasing the polymer concentration. The inverse of the correction function $1 / g^{t}$, ranging from 0 to 1 , is equivalent to $D_{\text {eff }}^{t} / D_{s}^{t}, \eta_{s} / \eta_{\text {eff }}^{t}$, or $f_{s}^{t} / f_{\text {eff }}^{t}$, in which the effective transport properties are compared with their corresponding values in solvent. The solid lines represent results for $\delta_{s}=0,0.2,0.5$, to $\delta_{s}=1$. When $\delta_{s}$ vanishes, $1 / g^{t}$ attains $\lambda$ or $\eta_{s} / \eta_{p}$; thus $\eta_{\text {eff }}$ $=\eta_{p}$. The deviation from the base line case $\left(\delta_{s}=0\right)$ increases 


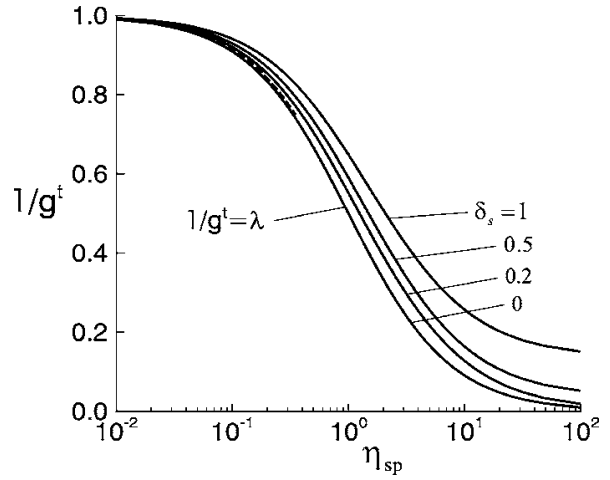

FIG. 5. The modified Stokes law, represented by the inverse of the correction function $1 / g^{t}$ versus the specific viscosity $\eta_{\mathrm{sp}}$, for a sphere in polymer solutions during long-time translational motion. The results shown are for various depletion thickness $\delta_{s}$. The dashed line refers to the asymptote given by Eq. (30) for $\delta_{s}=0.2$.

as $\delta_{s}$ increases. The reduction of friction as $\delta_{s}$ increases is also reflected on the apparent slip length $b^{t}$, approximated by Eq. (45). In the extreme case of the so-called "protein limit," $\delta_{s}$ approaches infinity and $g^{t}$ is unity for any specific viscosity.

Figure 6 shows the correction function $g^{r}$ predicted by Eq. (35) for the friction of a rotating sphere in a polymer solution versus the specific viscosity for various $\delta_{s}$ values. Comparing Figs. 5 and 6 for a given specific viscosity demonstrates a rotating sphere is more sensitive to the polymer depletion effect, estimated by the deviations from the baseline case with $\delta_{s}=0$. This behavior can be explained by the stronger first-order effect on the correction function formulated in Eq. (36) comparing to Eq. (30). In translational motion, the velocity field has a decaying rate proportional to $1 / r$, whereas it decays as $1 / r^{2}$ for the rotational case; thus it is reasonable to speculate that when a sphere rotates it only drives a thin layer of fluid immediately adjacent to the boundary in contrast to the translational case, which has a longer-range effect. Also in the rotational case the energy dissipates in a relatively small volume so that the transport behavior is only

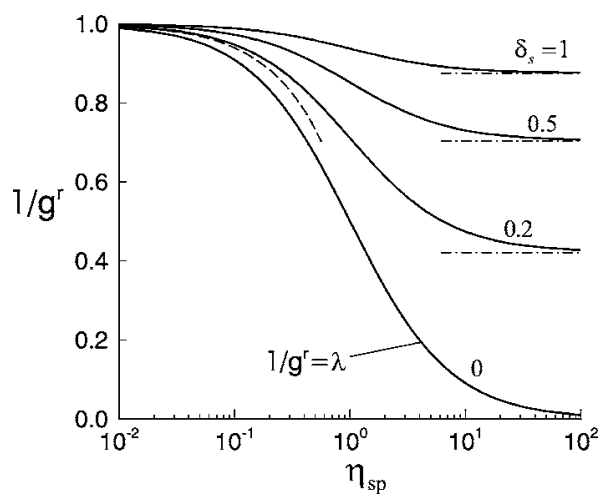

FIG. 6. The modified Stokes law, represented by the inverse of the correction function $1 / g^{r}$ versus the specific viscosity $\eta_{\mathrm{sp}}$, for a sphere in polymer solutions during long-time rotational motion with various depletion thickness $\delta_{s}$. The dashed line refers to the asymptote given by Eq. (36) for $\delta_{s}=0.2$. Dash-dotted lines are the results of Koenderink and co-workers [17]. sensitive to the physical properties of the fluid element near the surface. The dash-dotted lines in Fig. 6 correspond to the theoretical prediction of Koenderink et al. [17]:

$$
g^{r}\left(\delta_{s}\right)=\frac{\left(1+\delta_{s}\right)^{3}}{\left(1+\delta_{s}\right)^{3}-1},
$$

which appears to be the limiting case of our result, Eq. (35), with $\lambda \rightarrow 0$. Equation (47) holds for the cases with large polymer concentrations $\left(\eta_{p} \gg \eta_{s}\right)$ or for the short-time rotational motion such that the polymer segments may be treated as a fixed network instead of a movable suspension.

Experimentally, the translational friction $6 \pi \eta_{s} g^{t} a$ of diluted colloidal spheres can be measured by dynamic light scattering or direct monitoring of the sedimentation velocity of diluted settling colloidal spheres. The rotational friction $8 \pi \eta_{s} g^{r} a^{3}$ can be measured using depolarized dynamic light scattering or phosphorescence spectroscopy [46]. We compare our results with the experimental work of Koenderink and co-workers [17] who conducted a systematic study of the sedimentation, short-time translational, and short-time rotational diffusion on transparent colloidal fluorocarbon spheres through a polymer solution containing the polysaccharide xanthan. These data include the polymer concentration effect for both translational and rotational motions using a wellcharacterized sample. From phase transition studies, it has also been demonstrated that xanthan does not adsorb onto the colloidal spheres [47]. The approximated radius of gyration of the xanthan is $264 \mathrm{~nm}$, being a relatively stiff polysaccharide with a persistence length of $120 \mathrm{~nm}$. The standard expressions for a semiflexible chain thus effectively consists of about eight segments. For such semiflexible chains the effective depletion layers in dilute polymer solutions can be estimated [48] and for xanthan mixed with $93 \mathrm{~nm}$ spheres it follows that the depletion thickness of a dilute solution $\delta_{s 0}$ is approximately $123 \mathrm{~nm}$. The concentration dependence of the depletion thickness can be described by

$$
\delta_{s}^{-2}=\delta_{s 0}^{-2}+\xi^{-2}
$$

due to Fleer et al. [37], where the correlation length $\xi$ decreases with the polymer concentration $c[17,49]$, given by

$$
\xi=R_{g}\left(c / c^{*}\right)^{-3 / 4} \text {. }
$$

The overlap concentration of polymers $c^{*}$ was estimated to be 0.008 wt. $\%$.

In Fig. 7, we compare the theoretical predictions (solid lines) with the experimental results of Koenderink and coworkers [17]. The concentration dependent specific viscosity of xanthan solution at low shear condition can be described by the Martin equation [50],

$$
\eta_{\mathrm{sp}}=\lambda^{-1}-1=c[\eta] e^{k c[\eta]}
$$

where $[\eta]$ is the intrinsic viscosity, $6.25 \mathrm{~m}^{3} / \mathrm{kg}$, for the xanthan solution and the Huggins coefficient $k$ is 0.46 [17]. In Fig. 7, the asterisks are experimental data for $\lambda$ versus xanthan concentration $c$ and the dash-dotted line is the best fit using Eq. (50). Once the concentration is determined, $\eta_{\mathrm{sp}}, \lambda$, and $\delta_{s}$ can be obtained by Eqs. (48)-(50). The translational result $g^{t}$ predicts a reasonable trend for the measured sedi- 


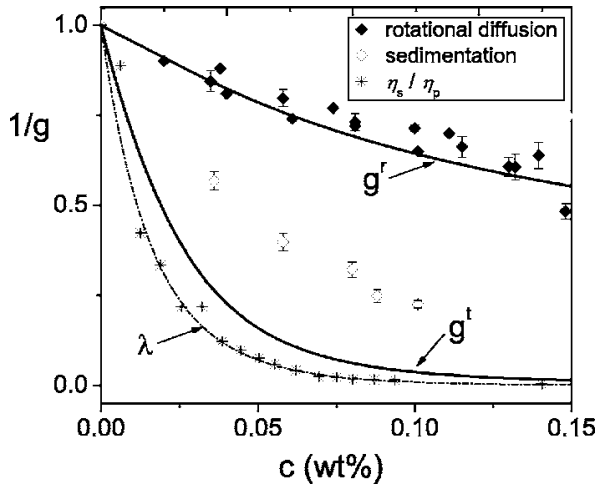

FIG. 7. Theoretical predictions (solid curves) of effective longtime rotational $\left(g^{r}\right)$ and translational $\left(g^{t}\right)$ motions of a colloidal sphere with radius $93 \mathrm{~nm}$ versus xanthan concentration $c$. Data points are experimental results measured by Koenderink and coworkers [17]. The translational motion was measured by the sedimentation (open diamonds) and the rotational motion was measured by the depolarized dynamic light scattering method (closed diamonds). Asterisks represent the measured viscosity $\eta_{s} / \eta_{p}$ for the xanthan solution versus the polymer concentration. Dash-dotted line is the best fit of the data points using Eq. (50).

mentation data. However, the model overpredicts $g^{t}$, meaning the sedimenting sphere experiences a smaller effective viscosity. The deviation may be caused by the simplified two-layer approximation that underpredicts the thickness of the depletion layer, or, by a more complicated scenario involving distortion of the polymer depletion layer due to the convective effect. Note that Koenderink et al. [17] also measured short-time translational motion using dynamic light scattering method, showing that the short-time behavior yields a much smaller $g^{t}$ than the sedimentation data and our prediction for the long-time translational motion. On a shorttime scale the sphere mainly moves through the surrounding depletion layer during its translational motion, whereas the long-time diffusion behavior we calculated is based on the total hydrodynamics resistance for the sphere to translate through the polymer solution. For translation motion, the fluid element at a longer distance away from the sphere can still sense the viscous force induced by the moving sphere, thus from either energy or momentum conservation point of view, a higher drag force is expected for the long-time case; see recent first principle simulation results by Krüger [51]. The predicted $g^{r}$ for the rotational self-diffusion is in excellent agreement with the measured data. Although our calculation is valid for the long-time rotational motion, experimental results show that the correction for both long-time and short-time rotational diffusions are similar, so that the model can be used for both conditions. The long-time rotational motion has a velocity decaying rate proportional to $1 / r^{2}$, meaning the sphere mainly moves around the depletion layer and is dynamically similar to the short-time rotational motion.

\section{CONCLUSIONS}

We present analytical results for the long-time friction experienced by a sphere that moves through a solution containing nonadsorbing chains by solving creeping flow equations. The hydrodynamics problem with variable viscosity was simplified by considering a symmetric depletion layer around the sphere where the viscosity is that of the solvent. Outside the depletion layer the viscosity equals the polymer solution viscosity. The non-Stokes-Einsteinian transport properties were analytically quantified and compared with the experimental data. We found that the rotational diffusion is not sensitive to the time scale, whereas translational diffusion is. For a system with a thin polymer depletion layer, the slip boundary condition can be used to characterize the transport behavior corresponding to the depletion effect.

\section{ACKNOWLEDGMENTS}

The authors thank G. Nägele and O. I. Vinogradova for helpful discussions at the early stage of this project. They acknowledge M. G. H. Krüger for sending an interesting M.Sc. thesis. They are indebted to G. H. Koenderink for providing experimental data.
[1] S. B. Zimmerman and A. P. Minton, Annu. Rev. Biophys. Biomol. Struct. 22, 27 (1993).

[2] T. H. Lin and G. D. J. Phillies, J. Phys. Chem. 86, 4073 (1982).

[3] G. S. Ullmann, K. Ullmann, R. M. Lindner, and G. D. J. Phillies, J. Phys. Chem. 89, 692 (1985).

[4] C. N. Onyenemezu, D. Gold, M. Roman, and W. G. Miller, Macromolecules 26, 3833 (1993).

[5] D. Gold, C. Onyenemezu, and W. G. Miller, Macromolecules 29, 5700 (1996).

[6] E. Donath, A. Krabi, M. Nirschl, V. M. Shilov, M. I. Zharkikh, and B. Vincent, J. Chem. Soc., Faraday Trans. 93, 115 (1997).

[7] C. W. Hoogendam, J. C. W. Peters, R. Tuinier, A. de Keizer, M. A. Cohen Stuart, and B. H. Bijsterbosch, J. Colloid Interface Sci. 207, 309 (1998).
[8] G. H. Koenderink, Ph.D. thesis, Utrecht University, Utrecht, (2003).

[9] D. Langevin and F. Rondelez, Polymer 19, 875 (1978).

[10] X. Ye, P. Tong, and L. J. Fetters, Macromolecules 31, 5785 (1998).

[11] P. Bartlett et al., Faraday Discuss. 123, 401 (2003).

[12] T. G. Mason and D. A. Weitz, Phys. Rev. Lett. 74, 1250 (1995).

[13] J. C. Crocker, M. T. Valentine, E. R. Weeks, T. Gisler, P. D. Kaplan, A. G. Yodh, and D. A. Weitz, Phys. Rev. Lett. 85, 888 (2000).

[14] L. Starrs and P. Bartlett, Faraday Discuss. 123, 323 (2003).

[15] T. A. Waigh, Rep. Prog. Phys. 68, 685 (2005).

[16] W. Brown and R. Rymdén, Macromolecules 21, 840 (1988).

[17] G. H. Koenderink, S. Sacanna, D. G. A. L. Aarts, and A. P. 
Philipse, Phys. Rev. E 69, 021804 (2004).

[18] J. K. G. Dhont, An Introduction to Dynamics of Colloids (Elsevier, Amsterdam 1996).

[19] K. Kang, J. Gapinski, M. P. Lettinga, J. Buitenhuis, G. Meier, M. Ratajczyk, J. K. G. Dhont, and A. Patkowski, J. Chem. Phys. 122, 044905 (2005).

[20] K. Kang, A. Wilk, J. Buitenhuis, A. Patkowski, and J. K. G. Dhont, J. Chem. Phys. 124, 044907 (2006).

[21] R. I. Cukier, Macromolecules 17, 252 (1984).

[22] K. L. Ngai and G. D. J. Phillies, J. Chem. Phys. 105, 8385 (1996).

[23] T. Odijk, Biophys. J. 79, 2314 (2000).

[24] Y. E. Solomentsev and J. L. Anderson, Phys. Fluids 8, 1119 (1996)

[25] S. Asakura and F. Oosawa, J. Polym. Sci. 33, 183 (1958).

[26] A. Vrij, Pure Appl. Chem. 48, 471 (1976).

[27] H. De Hek and A. Vrij, J. Colloid Interface Sci. 84, 409 (1981).

[28] J. F. Joanny, L. Leibler, and P. G. de Gennes, J. Polym. Sci., Polym. Phys. Ed. 17, 1073 (1979).

[29] P. G. de Gennes, Scaling Concepts in Polymer Physics (Cornell University Press, Ithaca 1979).

[30] E. Eisenriegler, J. Chem. Phys. 79, 1052 (1983).

[31] R. Tuinier and T. Taniguchi, J. Phys.: Condens. Matter 17, L9 (2005).

[32] P. G. de Gennes, C. R. Seances Acad. Sci., Ser. B 288, 219 (1979).

[33] P. G. de Gennes, Langmuir 18, 3413 (2002).

[34] L. Léger, J. Phys.: Condens. Matter 15, S19 (2003).

[35] C. Neto, D. R. Evans, E. Bonaccurso, H.-J. Butt, and V. S. J. Craig, Rep. Prog. Phys. 68, 2859 (2005).
[36] V. G. Levich, Physicochemical Hydrodynamics (Prentice-Hall, Englewood Cliffs, NJ, 1962).

[37] G. J. Fleer, A. M. Skvortsov, and R. Tuinier, Macromolecules 36, 7857 (2003).

[38] R. Tuinier, G. A. Vliegenthart, and H. N. W. Lekkerkerker, J. Chem. Phys. 113, 10768 (2000).

[39] R. Tuinier and H. N. W. Lekkerkerker, Eur. Phys. J. E 6, 129 (2001)

[40] H. Lamb, Hydrodynamics (Dover, New York 1945).

[41] J. Happel and H. Brenner, Low Reynolds Number Hydrodynamics (Prentice-Hall, New York 1965).

[42] L. D. Landau and E. M. Lifshitz, Fluid Mechanics, Course of Theoretical Physics Vol. 6 (Butterworth-Heinemann, Oxford 1987).

[43] G. G. Stokes, Trans. Cambridge Philos. Soc. 9, 8 (1851)

[44] P. G. de Gennes, Rev. Mod. Phys. 57, 827 (1985).

[45] A. B. Basset, A Treatise on Hydrodynamics, (Dover, New York 1961), Vol. 2.

[46] G. H. Koenderink, H. Zhang, D. G. A. L. Aarts, M. P. Lettinga, A. P. Philipse, and G. Nägele, Faraday Discuss. 123, 335 (2003).

[47] G. H. Koenderink, D. G. A. L. Aarts, V. W. A. de Villeneuve, A. P. Philipse, R. Tuinier, and H. N. W. Lekkerkerker, Biomacromolecules 4, 129 (2003).

[48] R. Tuinier, Eur. Phys. J. E 10, 123 (2003).

[49] D. Ausserré, H. Hervet, and F. Rondelez, Phys. Rev. Lett. 54, 1948 (1985).

[50] M. Tirrell, in Rheology, edited by C. W. Macosko (VCH Publishers, Minnesota 1994), Chap. 11.

[51] M. G. H. Krüger, M.Sc. thesis, University of Stuttgart, Germany 2006. 